\title{
Research article \\ Estimation of genotype MTBDRPLUS line probe assay in detection of rifampicin and isoniazid resistance in comparison to liquid culture (BACTEC-960) drug susceptibility testing in a tertiary care hospital from Eastern India
}

\author{
Chandan Kumar Poddar ${ }^{1}$, Namrata Kumari ${ }^{2}$, Rakesh Kumar ${ }^{3}$, Shivendra Kumar Shahi ${ }^{4}$, Shailesh Kumar ${ }^{6}$, Naresh \\ Kumar $^{5}$ \\ ${ }^{1} \mathrm{PhD}$ Research Scholar, ${ }^{2}$ Professor, ${ }^{3}$ Associate Professor, ${ }^{4}$ Professor, ${ }^{6}$ Additional Professor, Department of Microbiology, \\ ${ }^{5}$ Professor, Department of Medicine Indira Gandhi Institute of Medical Sciences, Patna, Bihar, India
}

(Received: March $2021 \quad$ Revised: June $2021 \quad$ Accepted: June 2021)

Corresponding author: Rakesh Kumar. Email: Chandan_microbiology@yahoo.co.in

\begin{abstract}
Introduction and Aim: India has the uppermost trouble of Multidrug resistant tuberculosis (MDR-TB) is a major challenge controlling resistance, reducing transmission and improving handling outcomes in MDR-TB patients is dependent on susceptibility testing. Isoniazid (INH) and rifampicin (Rif) are the key first-line antituberculosis drugs, and resistance to these drugs i.e., MDR-TB, is likely to result in treatment failure and poor clinical outcomes. The present study was done to compare the performance of line probe assay test (GenoType ${ }^{\circledR}$ MTBDRplus) with liquid culture (MGIT 960) system for the detection of resistance to first-line drugs.
\end{abstract}

Materials and Methods: We estimate the performance of LPAs to BACTEC MGIT 960 system for susceptibility testing of bacterial resistance to first-line drugs: rifampicin (RIF), isoniazid (INH).

Results: We performing Drug susceptibility testing (DST), 219/258 MTB cultures were viable after subculture the results of DST using the MGIT 960 system were compared to those obtained by line probe assay. LPA detected a total 46/258 (17.81\%) samples as drug resistant, of which 35/258 (13.70\%) were resistant to both rifampicin and isoniazid (MDR), 6/258 (2.28\%) were rifampicin mono-resistant samples and 11/258 (4.11\%) were isoniazid mono-resistant. Out of the culture-positive samples (219), LPA detected 39/219 (17.83\%) as drug-resistant, of which 31/219 (14.2\%) were resistant to both rifampicin and isoniazid, 5/193 (2.08\%) were rifampicin mono-resistant and 8/219 (3.7\%) were isoniazid mono-resistant.

Conclusion: Drug resistant TB poses an enormous threat to TB control programs worldwide. Effective treatment of MDR-TB is very expensive, particularly in middle income countries such as India.

Keywords: GenoType MTBDRplus test; BACTEC MGIT 960; MDR-TB; Rifampicin (RIF); Isoniazid (INH).

\section{INTRODUCTION}

$\mathrm{T}$ uberculosis (TB) residue a most important global health problem, an estimated 86 million individuals developed TB and 1.3 million died from the infection (1). With a population of around 1.24 billion, India is the largest country in the South East Asian region. It ranks first among the high TB burden countries which contributed $26 \%$ of the estimated global incident TB cases in 2012 and also among the 27 multidrug-resistant TB (MDR-TB) high-burden countries worldwide, contributing to $21 \%$ of all MDR-TB cases estimated among notified cases (2). Multi-Drug Resistant TB (MDR-TB) is distinct as resistance to, at slightest, isoniazid and rifampicin among first-line drugs, while Extensively Drug Resistant TB (XDR-TB) refers to MDR-TB with further resistance to, at slightest, any fluoroquinolone and a few one of second-line inject able drugs in calculation to isoniazid and rifampicin.

BACTEC MGIT 960, a rapid liquid phenotypic DST method, is used in frequent laboratories to test for resistance to first-line drugs. The MGIT preparation normally information drug susceptibilities according to predefined algorithms 4 to 13 days after inoculation (3). In 2008, WHO approved the use of the molecular test GenoType ${ }^{\circledR}$ MTBDR plus (Hain Lifescience, Nehren, Germany) for rapid revealing of high-risk MDR-TB cases constantly preparatory specimens $(4,5)$. The GenoType MTBDR plus assay is a commercially available Line Probe Assay (LPA) that allow specific gene markers associated with RIF and INH resistance to be detected.The GenoType ${ }^{\circledR}$ MTBDRplus evaluation is a molecular PCR-based extension and reverse blotting evaluate that employs accurate probes hybridized to nitrocellulose strips to recognize tuberculosis and its resistance to rifampicin (RIF) and isoniazid (INH) drugs (6). The approximation detects mutations in the rpoB gene for RIF resistance, in the katG gene for high-level INH resistance and in the inhA regulatory region gene for low-level INH resistance (3). During this study was appearance of molecular line probe assay to a MGIT 960 system for the detection of resistance to Anti-TB drugs. 


\section{MATERIALS AND METHODS}

\section{Study Design}

This was a prospective study in Department of Microbiology, Indira Gandhi Institute of Medical Sciences, Patna and Associated Hospital of Bihar, India between September 2018 and October 2020 were integrated. All tuberculosis isolates from sputum samples of clinically suspected cases of tuberculosis were seen in the study. The study protocol was approved by the IGIMS ethics committee (IEC numbe-485/IEC/2018).

\section{MGIT subculture and DST}

A whole volume of $200 \mu \mathrm{l}$ of MGIT 960 TB culture was being inoculated in the MGIT 960 vials for sub culturing and growth detection. Positive cultures will be stained using the Ziehl-Neelsen (ZN) staining method to confirm M. tuberculosis.

\section{Inclusion criteria}

Multidrug-resistant TB (MDR-TB) and pre-MDR isolates ii) Patient of 18 year to 75 year of age groups iii) both sexes (male and female).

\section{Exclusion criteria}

Other than Pulmonary Tuberculosis (PTB) and Extra Pulmonary Tuberculosis (EPTB).

\section{DNA isolation and line probe assay}

For LPA DST, isolate were all the samples subjected to LPA Mention the culture medias being used for the 1st line LPA.

\section{Agar proportion method}

We lined out discrepancies between LPA and MGIT liquid culture by behavior DST with the agar proportion technique on the Microplate (Lasec, SA) from Lowenstein-Jensen medium culture (7). A deferment of 5-10 mg of drug resistant-TB culture was used to make a McFarland standard.

\section{Specimen collection}

Sputum sample was being collected from PTB suspected patients, who was being attended hospital OPD. The Standard RNTCP protocol [8] was being followed for sample collection. The patients were provided a clean, dry, sterile wide-neck, leak-proof plastic container. Patients were being educated to cough deeply to produce sputum specimen and how to collect sputum without contaminating the collection container (8).

\section{Processing of samples}

Microscopic observation: Direct smear was being prepared from specimen for $\mathrm{ZN}$ staining and it will be examined under binocular bright field microscope, as per the standard procedure (8).

\section{Conventional drug susceptibility testing using BD} BBL $^{\text {TM }}$ MGIT $^{\text {TM }}$ AST SIRE SYSTEM

Subsequent to the cultures become positive, they were experienced for DST according to the BD BBL $^{\mathrm{TM}}$ MGIT $^{\mathrm{TM}}$ AST SIRE system within 7 days. For every sample, $4 \mathrm{ml}$ of Middlebrook $7 \mathrm{H} 9$ broth was deposit in a sterilized universal bottle with 8-10 glass beads. A $10 \mu \mathrm{l}$ sterile inoculating loop was used to abrade colonies from the LJ medium. The colonies were hanging in the broth to a turbidity of 1.0 McFarland standard. The deferment was vortexed for 2-3 min to break the large colonies. The suspension was then permitted to sit for $20 \mathrm{~min}$ to let the sediment settle at the bottom of the tube. The supernatant was suspiciously transfer to another sterile universal bottle and allowed to sit for another $20 \mathrm{~min}$. The supernatant was then transfer to a third sterile universal bottle and adjusted to $0.5 \mathrm{McF}$ arland. The adjustment was done by comparing the turbidity of the suspension with the turbidity of the McFarland turbidity standard made by combining $1 \%$ sulfuric acid $\left(\mathrm{H}_{2} \mathrm{SO}_{4}\right)$ and $1 \%$ anhydrous barium chloride $\left(\mathrm{BaCl}_{2}\right)$ in the following volumes: for $1.0 \mathrm{McF}$ arland (9.95 $\mathrm{ml} \mathrm{H}_{2} \mathrm{SO}_{4}$ and $0.05 \mathrm{ml} \mathrm{BaCl}_{2}$ ) and for 0.5 McFarland $\left(9.9 \mathrm{ml} \mathrm{H}_{2} \mathrm{SO}_{4}\right.$ and $0.1 \mathrm{ml} \mathrm{BaCl}_{2}$ ). For susceptibility testing, $1 \mathrm{ml}$ of the 0.5 McFarland-adjusted deferment be diluted in $4 \mathrm{ml}$ of sterile saline to make 1:5 dilution $(9,10)$.

\section{Statistical analysis}

Sensitivity, specificity, positive predictive value (PPV), and negative predictive value (NPV), based on conformity between the DST gold standard and the index assay and 95\% confidence intervals (95\% CIs), were considered for both the antibiotic class and individual drugs. The agreement, sensitivity, specificity, positive and negative predictive values of LPA compared to BACTEC MGIT 960 system were intentional for Isoniazid (INH), Rifampicin (RIF).

\section{RESULTS}

Two hundred and eighty-two sputum positive samples of patients suspected to have drug resistant TB were picked and subjected to LPA and Culture MGIT. MGIT was positive for M. tuberculosis in 258/282(91.48\%) were AFB smear positive.

\section{Socio demographic characteristics}

Of the 258 samples, 90 (34.80\%) were from female and $168(65.20 \%)$ from male participants. Transmission of tuberculosis is influenced by gender. Tuberculosis is more common among males than females (CDC, 2016; WHO, 2016) [17]. The prevalence of tuberculosis among males 168 $(65.20 \%)$ was higher than that of females 90 (34.80\%) and male to female ratio (M: F) was 1.7 $(168 / 90)$, this difference was highly significant $(\mathrm{p}<0.01)$. 
Table 1: Distribution of TB patients according to gender

\begin{tabular}{|c|c|c|}
\hline \multirow{2}{*}{ Gender } & \multicolumn{2}{|c|}{$\begin{array}{c}\text { Culture Based Diagnosis } \\
\text { on Liquid culture }\end{array}$} \\
\cline { 2 - 3 } & + Ve & $\%$ \\
\hline Male & 168 & 65.20 \\
\hline Female & 90 & 34.80 \\
\hline Total & 258 & $100 \%$ \\
\hline Chi-square- $\chi 2$ & - & 21.6791 \\
\hline P-value & - & 0.00001 \\
\hline
\end{tabular}

To evaluate the performance of LPA on smear positive results, 258 samples were used by LPA detected 241/282 (85.46\%) samples as positive. LPA reported sensitivity and specificity of 99.31 and $97.43 \%$ respectively with positive and negative predictive value of $99.31 \%$ and $98.70 \%$ respectively. Of 282 samples tested, Culture MGIT detected $M$. tuberculosis in 219 (77.66\%) samples, LPA detected M. tuberculosis in 241/282 (85.46\%). Using Culture MGIT as the gold standard, the LPA correctly recognized M. tuberculosis in 216 of 219 Culture MGIT positive samples.

\section{Drug susceptibility testing (DST)}

MGIT culture positive for M. tuberculosis in 219/258 $(84.88 \%)$ were AFB smear positive. Of MGIT cultures, $6 / 258(2.32 \%)$ were contaminated, and due to site/lab error 8 had the X200 error, and 12 had the X400 error. The remaining Two hundred nineteen (219) samples were analysed.

Drug susceptibility testing against first line drugs which used for TB treatment was carried out on 219 sputum culture positive isolates. (Table 1). The results of the routine DST using the MGIT 960 system were compared to the results obtained by LPA. Drug-resistance isolates were distributed as follows: $29(13.24 \%)$ isolates were resistant to one drug (mono drug resistance) including 5 (2.28\%) isolates resistant to RIF, $6(2.74 \%)$ to $\mathrm{INH}, 14$ (6.39\%) to STP and 4(1.83\%) to EMB. 96 (43.84\%) isolates were multidrug resistance (MDR) of which $12(5.48 \%)$ isolates were resistant against both INH and RIF, 55 (26.11\%) were drug resistant against all four drugs, while $27(12.33 \%)$ isolates were resistant to different combinations of three drugs. Finding of MDR-TB isolates to combinations of 3 and 4 drugs may point to presence of XDR - TB. Poly drug resistance against two or three drugs was observed in $17(7.76 \%)$ isolates. The results also showed the highest prevalence of resistant to any drug was identified against INH (51.59\%), followed by STP, RIF and EMB (46.58\%, 45.21\%, and $33.79 \%)$ respectively.

\section{First line DST}

Table 2: Results of drug susceptibility test in first line anti-Tb drugs

\begin{tabular}{|c|c|c|c|}
\hline \multicolumn{2}{|c|}{ Category, Number and Percentage of Isolates } & Drug & Frequency $(\%)$ \\
\hline \multicolumn{2}{|c|}{ Susceptible to all drugs } & $\mathrm{INH}+\mathrm{STP}+\mathrm{RIF}+\mathrm{EMB}$ & $78(35.62 \%)$ \\
\hline \multirow{15}{*}{ Resistant 141 (64.38\%) } & \multirow{4}{*}{$\begin{array}{c}\text { Monodrug } \\
\text { resistance } \\
29(13.24 \%)\end{array}$} & RIF & $5(2.28 \%)$ \\
\hline & & INH & $6(2.74 \%)$ \\
\hline & & STP & $14(6.39 \%)$ \\
\hline & & EMB & $4(1.83 \%)$ \\
\hline & \multirow{4}{*}{$\begin{array}{c}\text { Multidrug } \\
\text { resistance } \\
96(43.84 \%)\end{array}$} & INH + RIF & $12(5.48 \%)$ \\
\hline & & INH + RIF+EMB & $27(12.33 \%)$ \\
\hline & & $\mathrm{INH}+\mathrm{RIF}+\mathrm{STP}$ & \\
\hline & & $\mathrm{INH}+\mathrm{STP}+\mathrm{RIF}+\mathrm{EMB}$ & $57(26.11 \%)$ \\
\hline & \multirow{7}{*}{$\begin{array}{l}\text { Polydrug } \\
\text { resistance } \\
16(7.30 \%)\end{array}$} & RIF+EMB & \multirow[t]{7}{*}{$16(7.30 \%)$} \\
\hline & & RIF+STP & \\
\hline & & RIF+STP+EMB & \\
\hline & & $\mathrm{INH}+\mathrm{STP}+\mathrm{EMB}$ & \\
\hline & & EMB+STP & \\
\hline & & INH+EMB & \\
\hline & & INH+STP & \\
\hline \multicolumn{2}{|c|}{ Total } & ----------- & $219(100 \%)$ \\
\hline \multirow{4}{*}{\multicolumn{2}{|c|}{ Any resistance to: }} & INH & $113(51.59 \%)$ \\
\hline & & STP & $102(46.58 \%)$ \\
\hline & & RIF & $99(45.21 \%)$ \\
\hline & & EMB & $74(33.79 \%)$ \\
\hline \multicolumn{2}{|c|}{ Chi-square- $\chi 2$} & --- & 11.342 \\
\hline \multicolumn{2}{|c|}{ P-value } & --- & 0.0003 \\
\hline
\end{tabular}

INH= Isoniazid, STP = Streptomycin, RIF= Rifampicin, EMB= Ethambutal, Monodrug = Single drug resistance, Multidrug resistance $=$ resistance at least INH and RIF, Poly drug $=$ Resistance to two and more drugs without the combination of INH and RIF.

\section{Results of Line Probe Assay (LPA) test}

LPA detected a total $46 / 258(17.81 \%)$ samples as drug resistant, of which 35/258 (13.70\%) were resistant to both rifampicin and isoniazid (MDR), $6 / 258(2.28 \%)$ were rifampicin mono-resistant samples and $11 / 258 \quad(4.11 \%)$ were isoniazid 
mono-resistant. Out of the culture-positive samples (219), LPA detected 39/219 (17.83\%) as drug-resistant, of which $31 / 219 \quad(14.2 \%)$ were resistant to both rifampicin and isoniazid, 5/193 $(2.08 \%)$ were rifampicin mono-resistant and 8/219 (3.7\%) were isoniazid mono-resistant (Figure-1). When compared to MGIT liquid DST for finding of drug resistance, LPA accurately detected all samples resistant on MGIT liquid DST but disastrous to detect two samples of isoniazid mono-resistant and showed them as sensitive. Sensitivity and specificity for DR-TB recognition was found to be $95.83 \%$ (95\% CI: 80.8-99.3) and $99.53 \%$ (95\% CI: 97.7-100), respectively.

Table 3: Hybridization patterns obtained with the GenoType MTBDRsl assay

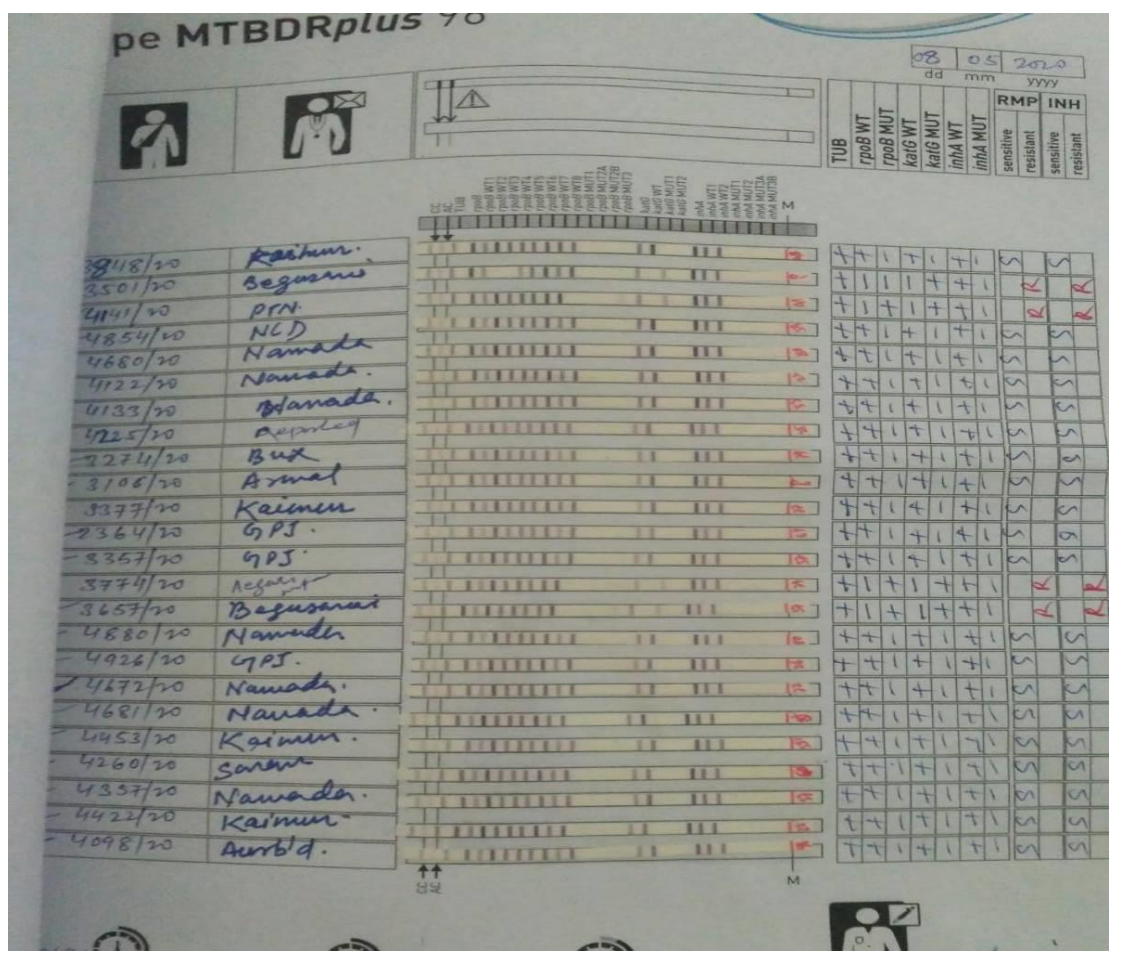

\section{DISCUSSION}

Early diagnosis and treatment halt the spread of drug resistant bacilli in the community but the current convent Effective treatment of MDR-TB is very expensive, particularly in middle income countries such as India. Early diagnosis and successful treatment require a sensitive and specific diagnostic tool. According to the WHO, a successful handling regimen depends on optimal susceptibility testing of Mycobacterium tuberculosis to anti-TB drugs (11). The precision of susceptibility testing result varies with the drug tested as well as with the DST method.

The present study was finished to evaluate two rapid molecular diagnostic tests for the diagnosis of drug resistance with the conventional gold standard method i.e., MGIT liquid culture DST. In this study was comparing the routine of the MGIT 960 system to line probe assay for the detection of drug susceptibility to first line drugs.

Our LPA result compares to the sensitivity and specificity of LPA investigative platform in India two studies showed a sensitivity and specificity of 96 and 99\% respectively in 248 smear positive patients (12) South African and South American was 81 and 100\% correspondingly (13) and, sensitivity and specificity of 68.4 and $89.3 \%$ correspondingly from 213 sputum smear negative samples (14).
In learning in New Delhi agreement sensitivity and specificity of 97.6 and $94.4 \%$ when they use LPA in smear positive samples (15) respectively to the South Africa study description a sensitivity and specificity of 97.7 and $91.8 \%$ respectively (16). In previous studies done in African countries, the sensitivity and specificity of Rifampicin mono resistance uncovering was 96.4 and $100 \%$ among smear positive samples and 77.8 and $98.2 \%$ among smear negative samples in Ethiopia and 100 and $96.1 \%$ among smear positive population in Uganda (17).

The current learning information a high consistency comparable to what was report in India, of $100 \%$ between MGIT960 and LPA results $(8,19)$ equally LPA reporting the same with the conventional DST at $96 \%$ in New Delhi (20).

\section{Limitations}

LPAs are as composite to achieve as conventional culture and DST methods and entail skilled and welltrained laboratory employees, as well as adequate laboratory space and design to reduce the risk of false-positive results. dispensation of smear-positive specimens for unswerving testing should be performed in a BSL-2 level laboratory, whereas performing LPAs on positive cultures requires BSL-3 laboratory infrastructure. 


\section{CONCLUSION}

TB control programmes are needed to ensure universal access to MDR-TB diagnosis and reduce the development and transmission of drug-resistant TB. We experiential enormous diagnostic presentation of the LPA, confirming its reliability and efficiency for the rapid diagnosis of MDR-TB. The current study performance sensitivity and specificity in detecting MDR-TB cases with a appreciably lesser turnaround time as compared to liquid culture DST method and instantaneous detection of Rif and INH resistance. This performance saves several days of time necessary for liquid culture and DST. Hence, a schedule use of LPA can considerably reduce the time to diagnose RIF and INH resistance and can potentially permit previous beginning of proper drug therapy, thereby facilitating prevention of further transmission of drug-resistant strains. In the background of rising drug resistance in MTB, the MTBDR plus assay may be a good tool in the diagnosis and management of MDR-TB.

\section{CONFLICT OF INTEREST: Authors declare no} conflict of interest.

\section{REFERENCES}

1. World Health Organization. Global Tuberculosis Report 2013. World Health Organization; 2013. Available from: http://www.who.int/htm/tb/2013.11.

2. World Health Organization. Annual Report. Tuberculosis Control in the South East Asia Region. World Health Organization; 2014. Available from: http://www.searo. who.int/tb/annual_tb_report_2014.pdf?ua=1.

3. Piersimoni, C., Olivieri, A., Benacchio, L., Scarparo, C. Current perspectives on drug susceptibility testing of Mycobacterium tuberculosis complex: the automated nonradiometric systems. J Clin Microbiol. 2006; 44: 20 -28.

4. Akpaka, P. E., Baboolal, S., Clarke, D., Francis, L., Rastogi, N. Evaluation of methods for rapid detection of resistance to isoniazid and rifampin in Mycobacterium tuberculosis isolates collected in the Caribbean. Journal of clinical microbiology. 2008 Oct; 46(10): 3426-3428.

5. Barnard, M., Albert, H., Coetzee, G., O'Brien, R., Bosman M. E. Rapid molecular screening for multidrug resistant tuberculosis in a high-volume public health laboratory in South Africa. Am. J. Respir. Crit. Care Med. 2008; 177787177792.

6. Huang, W. L., Chen, H. Y., Kuo, Y. M., Jou, R. Performance assessment of the GenoType MTBDR plus test and DNA sequencing in detection of multidrugresistant Mycobacterium tuberculosis. J Clin Microbiol. 2009; 47: 2520-2524. Doi: 10.1128/JCM.02499-08

7. Kent, P. T., Kubica, P. G. Public Health Mycobacteriology: a guide for the level III laboratory. Atlanta: U.S. Department of Health and Public Health Services, Centers for Disease Control; 1985.

8. Revised National Tuberculosis Control Programme. DOTSPlus Guidelines [Internet]. New Delhi: Central TB Division Directorate General of Health Services; 2010 January. Available from: health. bih.nic.in/Docs/Guidelines /Guidelines-DOTS-plus.pdf.

9. BD BBLTM MGITTM AST SIRE System. For the Antimycobacterial Susceptibility Testing of Mycobacterium tuberculosis" Available from: http://www. Legacy.bd.com/ds/ technical Center/inserts/8809591JAA (03). pdf. [Last accessed on 2018 Apr 03].
10. Dalynn Biological. McFarland Standard -For in vitro use only- Catalogue No. TM50-TM60. Available from: http://www.dalynn.com/dyn/ck_ assets/files/tech/TM53.pdf. [Last accessed on 2018 Apr 03].

11. World Health Organization. Global Tuberculosis Report 2016. Geneva: World Health Organization; 2016. World Health Organization, Geneva, Switzerland. http://www.who.int/tb /publications/global report/2016/en/.

12. Luetkemeyer, A. F., Kendall, M. A., Wu, X., Lourenço, M. C., Jentsch, U., Swindells, S., et al., Evaluation of two-line probe assays for rapid detection of mycobacterium tuberculosis, tuberculosis (TB) drug resistance, and non-TB mycobacteria in HIV-infected individuals with suspected TB. J Clin Microbiol. 2014; 52: 1052-1059. https:// doi.org /10.1128/JCM.02639-13.

13. Van Deun, A., Barrera, L., Bastian, I., Fattorini, L., Hoffmann, H., Kam, K. M., et al., Mycobacterium tuberculosis strains with highly discordant rifampin susceptibility test results. J Clin Microbiol. 2009; 47: 35013506.

14. Singh, B. K., Sharma, S. K., Sharma, R., Sreenivas, V., Myneedu, V. P., Kohli, M., et al., Diagnostic utility of a line probe assay for multidrug resistant-TB in smear negative pulmonary tuberculosis. PLoS One. 2017; 12: e0182988. https://doi. org/10.1371 / journal. pone.0182988.

15. Ritu, S., Jyoti, A., Prabha, L., Manpreet, B., Myneeedu, B. P., Digamber, B. Comparison of line probe assay with liquid culture for rapid detection of multi-drug resistance in Mycobacterium tuberculosis. Indian J Med Res. 2012; 136: 1044-1047.

16. Tomasicchio, M., Theron, G., Pietersen, E., Streicher, E., Stanley-Josephs, D., Van Helden, P., et al., The diagnostic accuracy of the MTBDRplus and MTBDRsl assays for drug-resistant TB detection when performed on sputum and culture isolates. Sci Rep. 2016; 6: 17850. https: //doi.org/10.1038/srep17850.

17. Albert, H., Bwanga, F., Mukkada, S., Nyesiga, B., Ademun, J. P., Lukyamuzi, G., et al., Rapid screening of MDR-TB using molecular line probe assay is feasible in Uganda. BMC Infect Dis. 2010; 10:41. https://doi.org/10.1186/14712334-10-41.

18. Rufai, S. B., Kumar, P., Singh, A., Prajapati, S., Balooni, V., Singh, S. Comparison of xpert MTB/RIF with line probe assay for detection of rifampin-monoresistant mycobacterium tuberculosis. J Clin Microbiol. 2014; 52: 1846-1852. https://doi. org/10.1128/JCM.03005-13.

19. Singh, M. N., Lal, A., Poddar, C. K., Ahmad, I., Efficacy of fine-needle aspiration technique, Ziehl-Neelsen stains and culture (BACTEC) in diagnosis of tuberculous lymphadenitis in tertiary care hospital, Gaya, India. J. Evid. Based Med. Healthc. 2017; 4(70): 4164-4169. DOI: 10.18410/jebmh/2017/829

20. Yadav, R. N., Singh, B. K., Sharma, S. K., Sharma, R., Soneja, M., Sreenivas, V., et al., Comparative evaluation of GenoType MTBDR plus line probe assay with solid culture method in early diagnosis of multidrug resistant tuberculosis (MDR-TB) at a tertiary Care Centre in India. PLoS One. 2013; 8: e72036. https:// doi.org /10.1371 /journal. pone. 0072036 . 